\title{
Langmuir
}

This document is confidential and is proprietary to the American Chemical Society and its authors. Do not copy or disclose without written permission. If you have received this item in error, notify the sender and delete all copies.

\section{Superposition of Translational and Rotational Motions under Self-Propulsion of Liquid Marbles Filled by Aqueous Solutions of Camphor}

\begin{tabular}{|r|l|}
\hline Journal: & Langmuir \\
\hline Manuscript ID & la-2017-03356m.R1 \\
\hline Manuscript Type: & Article \\
\hline Date Submitted by the Author: & $\mathrm{n} / \mathrm{a}$ \\
\hline Complete List of Authors: & $\begin{array}{l}\text { Bormashenko, Edward; Ariel University Faculty of Natural Sciences, Physics } \\
\text { Frenkel, Mark; Ariel University, } \\
\text { Bormashenko, Yelena; Ariel Iniversity, } \\
\text { Chaniel, Gilad; Ariel University, Physics Faculty } \\
\text { Valtsifer, Victor; Institute of Technical Chemistry, UB RAS } \\
\text { Binks, Bernard; University of Hull, Chemistry; University of Hull, }\end{array}$ \\
\hline
\end{tabular}




\title{
Superposition of Translational and Rotational Motions under Self-
}

\section{Propulsion of Liquid Marbles Filled by Aqueous Solutions of}

\section{Camphor}

\author{
Edward Bormashenko, ${ }^{*}$ Mark Frenkel, ${ }^{a}$ Yelena Bormashenko, ${ }^{a}$ Gilad Chaniel ${ }^{b}$, \\ Viktor Valtsifer ${ }^{\mathrm{c}}$ and Bernard P. Binks ${ }^{\mathrm{d}}$ \\ ${ }^{a}$ Ariel University, Chemical Engineering, Biotechnology and Materials Department, \\ P.O.B. 3, 40700, Ariel, Israel \\ ${ }^{b}$ Ariel University, Department of Physics, P.O.B. 3, 40700, Ariel, Israel \\ ${ }^{c}$ Institute of Technical Chemistry, UB RAS, Academician Korolev St., 3, 614013 \\ Perm, Russian Federation \\ ${ }^{d}$ School of Mathematics and Physical Sciences, University of Hull, \\ Hull. HU67RX. UK \\ ${ }^{*}$ Corresponding author:
}

Edward Bormashenko

Ariel University, Chemical Engineering, Biotechnology and Materials Department

P.O.B. 3, Ariel 40700, Israel

Phone: +972-3-906-6134

Fax: +972-3-906-6621

E-mail: edward@ariel.ac.il

Contains ESI (4 movies and the Fourier spectra of the time dependence of the translational velocity of a marble) 


\begin{abstract}
Self-locomotion of liquid marbles, coated with lycopodium or fumed fluorosilica powder, filled with a saturated aqueous solution of camphor and placed on a water/vapor interface is reported. Self-propelled marbles demonstrated a complicated motion, representing a superposition of translational and rotational motions. Oscillations of the velocity of the center of mass and the angular velocity of marbles, occurring in anti-phase, were registered and explained qualitatively. Self-propulsion occurs due to the Marangoni soluto-capillary flow inspired by the adsorption of camphor (evaporated from the liquid marble) by a water surface. Scaling laws describing translational and rotational motions are proposed and checked. The rotational motion of marbles arises from the asymmetry of the field of the Marangoni stresses due to the adsorption of camphor evaporated from a marble.
\end{abstract}

Keywords: liquid marbles; hydrophobic fluorosilica powder; lycopodium; camphor; gradient of surface tension; soluto-capillary driven locomotion. 


\section{INTRODUCTION}

The self-generated hydrodynamic and chemical fields may induce motion of particles or droplets placed at the liquid/vapor ${ }^{1-6}$ or solid/vapor ${ }^{7-11}$ interfaces. We consider the case when a non-stick water droplet, coated with hydrophobic particles, containing a volatile compound (which is camphor in our case) creates its own motion by using the gradient of the specific surface energy of the liquid (water) support. Selfpropulsion occurs under breaking of spherical symmetry of a non-stick droplet, taking place when evaporation of the volatile compound (camphor) in a certain direction is spontaneously increased. ${ }^{12}$ A volatile compound evaporated from a marble and consequently adsorbed by a water/vapor interface produces Marangoni solutocapillary flows, resulting in the self-propulsion of a droplet. ${ }^{13-16}$

In our study we used the so-called liquid marbles ${ }^{17-33}$, in other words, nonstick droplets coated with hydrophobic nano- or micro-sized particles filled with saturated aqueous solutions of camphor. It should be emphasized that the solid particles do not cover the surface of a droplet hermetically, thus, enabling evaporation of water and camphor from a marble. ${ }^{22,28,34}$ Also hydrophobic particles separate a droplet from a liquid (water) support, creating a pseudo-Leidenfrost contact situation, within which the drop is disconnected from the liquid support by its own vapor. ${ }^{35-41}$ The possibility to involve the pseudo-Leidenfrost contact of liquid marbles containing volatile compounds for their self-locomotion was reported recently. ${ }^{42,43}$ The connection of aqueous ethanol solutions filling a marble to the atmosphere gave rise to the self-propulsion of the marbles across a liquid surface. ${ }^{42,43}$ Light-induced Marangoni flows enabled transport of liquid marbles, as reported in ref. 44.

In the present work, the effect of self-propulsion is demonstrated for liquid marbles containing aqueous solutions of camphor. Dissolution of camphor, resulting 
in the self-locomotion of so called "camphor boats", is a well-known effect and the subject of intense research recently ${ }^{45-49}$. By contrast, in our investigation camphor is separated from a liquid support, and the effect of self-propulsion arises from its asymmetric evaporation from a liquid marble followed by adsorption at the water/ vapor interface. Floating of non-stick droplets was studied in detail recently in ref. 50. It was demonstrated that the floating state for relatively undeformable drops (such as liquid marbles) is sensitive to the relative tensions of the drop-vapor and drop-liquid interfaces. ${ }^{50}$ Self-propelled droplets give rise to numerous applications, including smart lab-on-chip systems ${ }^{51}$, optical probing ${ }^{52}$ and cell cultivation. ${ }^{53}$

\section{EXPERIMENTAL}

\subsection{Materials}

Liquid marbles containing aqueous solutions of camphor were manufactured with two kinds of powders, namely lycopodium and the extremely hydrophobic fumed fluorosilica powder. ${ }^{54}$ The primary diameter of fumed fluorosilica particles is 20-30 $\mathrm{nm}$ and they originate from hydrophilic silica (Wacker Chemie) after reaction with tridecafluoro-1,1,1,2-tetrahydrooctyltrimethoxysilane. ${ }^{54}$ The residual silanol content on their surfaces is $50 \%$ and the fluorine content is $10.9 \%$. Camphor $\left(\mathrm{C}_{10} \mathrm{H}_{16} \mathrm{O}, 96 \%\right)$ and lycopodium were supplied by Sigma-Aldrich. Lycopodium is the yellow pollen collected from the spores of a perennial evergreen plant (Lycopodium clavatum). This pollen possesses hierarchical structure and is strongly hydrophobic.

The outer diameter of lycopodium particles is $c a$. $50 \mu \mathrm{m}$ (for SEM images of lycopodium particles see ref. 55).

\subsection{Manufacture of liquid marbles}


Droplets containing saturated aqueous solutions of camphor (concentration $\left.c=1.2 \frac{g}{d^{3}}\right)$ were coated with lycopodium or fumed fluorosilica powder according to the protocol described in ref. 42. De-ionized water was prepared from a synergy UV water purification system from Millipore SAS (France). The specific resistivity of water was $\hat{\rho}=18.2 \mathrm{M} \Omega \times \mathrm{cm}$ at $25^{\circ} \mathrm{C}$.

Saturated aqueous solutions of camphor were filtered with $45 \mu \mathrm{m}$ polyvinylidene fluoride meshes, enabling removal of small non-dissolved camphor particles. Droplets containing saturated aqueous solutions of camphor of volume between 10 and $50 \mu \mathrm{L}$ were spread on a layer of lycopodium or fumed fluorosilica powder situated on a glass slide. The slide was vibrated slightly giving rise to the formation of liquid marbles. The marbles are not hermetically coated by powder; they evaporate as discussed in detail in refs. 25-28 and 34 and 35. The lifetime of marbles enabling observing of self-propulsion was established as $c a .2 \mathrm{~min}$.

\subsection{Registration of self-propulsion of liquid marbles}

The marbles were placed on the surface of de-ionized water and floated, as shown in Figure 1. The motion of liquid marbles was registered from above with a rapid camera (Casio EX-FH20). The linear and angular velocities and accelerations of self-propelled marbles were established by the frame-by frame analysis of their motion registered with the camera. For the registration of the rotational angle of marbles, denoted $\Psi$, particles of carbon black were placed on the surface of a marble which served as the marker. All the experiments were performed at ambient temperature of $23-25^{\circ} \mathrm{C}$.

2.4. Study of the influence of the camphor evaporated from marbles on the surface tension of the water support 
For the study of the impact exerted by camphor evaporated from the droplet and condensed on the surface tension of the water support the following experiments were carried out. The change in the surface tension of a pendant de-ionized water droplet was measured within the protocols described in detail in ref. 56. According to the first protocol, a water droplet was suspended over the aqueous camphor solution/vapor interface, and the surface tension was measured as a function of the distance $h$ between the water surface and the bottom of the droplet (see Figure 2). Under the second protocol, a water droplet was placed at some constant height $h$ above the surface of the saturated aqueous solution of camphor, and the kinetics of the surface tension change was measured. Surface tension was measured with the pendant droplet method using the Ramé-Hart Advanced Goniometer Model 500-F1 at ambient conditions. The initial volume of pendant water droplets was 5-6 $\mu \mathrm{L}$.

Figure 3 presents the dependence of the surface tension on the separation of a pendant droplet from the surface of the saturated aqueous solution of camphor. The surface tension was measured for the same water droplet when it moved down from 10 to $1 \mathrm{~mm}$ distance $h$ above the puddle of the aqueous camphor solution. The value of the surface tension decreased from $72 \frac{\mathrm{mJ}}{\mathrm{m}^{2}}$ to $68 \frac{\mathrm{mJ}}{\mathrm{m}^{2}}$. Thus, the maximal jump of the surface tension (denoted $\Delta \gamma$ ) due to the evaporation of camphor followed by its adsorption at the pendant droplet/vapor interface was established as $c a .4 .0 \frac{\mathrm{mJ}}{\mathrm{m}^{2}}$. The same value of the maximal change in the surface tension of a pendant water droplet was also established for water droplets placed at the fixed height $h$ above the surface of the saturated aqueous solution of camphor when the kinetics of the change in the surface tension of pendant droplet was measured.

\subsection{Thermal imaging of self-propulsion}


Thermal imaging of the self-propulsion was monitored with a Therm-App TAS19AQ-1000-HZ thermal camera (Opgal Optronic Industries, Karmiel, Israel) equipped with a LWIR (long wavelength infrared) $6.8 \mathrm{~mm} \mathrm{f} / 1.4$ lens (see the Supplementary Material).

\subsection{Spectral analysis of the time dependencies of the kinematic parameters of} the motion

The Fourier analysis of the time dependencies of linear and angular velocities of liquid marbles was performed with the software Excel.

\section{RESULTS AND DISCUSSION}

\subsection{Superposition of translational and rotational motion of self-propelled marbles}

Liquid marbles containing aqueous camphor solutions were placed on the surface of de-ionized water contained in a Petri dish (diameter $78 \mathrm{~mm}$ ) as described in the Experimental section. The marbles demonstrated a kind of self-propulsion similar but somewhat different from that described in refs. 42 and 43, in which marbles filled by aqueous solutions of alcohols were studied. The marbles filled by aqueous solutions of alcohols started their self-propelled motion immediately after being placed on a water support. ${ }^{42,43}$ In contrast, liquid marbles filled with saturated aqueous solutions of camphor were first slowed down (the initial velocity supplied to a marble after being placed on the water support decayed to zero) and often even stayed at rest during $c a$. 1-10 s and started their self-propulsion after the aforementioned waiting interval. The entire time span of the self-propulsion was $c a .2 \mathrm{~min}$. Such a character of motion was convenient for investigation, due to the elimination of artifacts arising from the initial velocity and acceleration of marbles put at the water/vapor interface. 
The superposition of translational and rotational motions of marbles described schematically in Figure 4 was observed (see also the Supplementary Material). In parallel with the translational displacement, marbles rotated about the vertical axis passing through the center of mass. The overall time span of the translational motion accompanied by rotation was $c a .1 \mathrm{~min}$.

We first inspect the mechanism of self-propulsion qualitatively. Obviously, self-propulsion demands breaking of the initial spherical symmetry of marbles. Consider the spontaneous increase in evaporation of volatile camphor from the marble in the direction of $-x$, as depicted in Figure 5 (it should be emphasized that liquid marbles are not coated hermetically by a powder coating ${ }^{27,28}$ ). This increase will give rise to the Marangoni soluto-capillary flow, resulting in the force $\vec{F}$ (shown by the green arrow in Figure 5) driving the marble in the direction of $x$. In parallel it develops a fascinating instability transporting marbles. ${ }^{42,43}$ The displacement of marbles in turn enhances the evaporation, withdrawing the camphor vapor from the layer separating the marble from the supporting liquid. ${ }^{43}$

Assume that the rotational motion of a marble is planar, namely all of the particles constituting a marble move parallel to a liquid/vapor interface (this assumption coincides with the experimental observations, see the Supplementary Material). Thus, the general model describing the self-propulsion is represented by Eq. 1. The Marangoni soluto-capillary induced motion ${ }^{12-14,41,42,53-60}$ can be described by the system of Newtonian equations as follows:

$$
\begin{aligned}
m \frac{d \vec{v}_{c m}}{d t} & =\vec{F}_{f r}+\alpha a^{2} \nabla \gamma=-\chi a \eta \vec{v}_{c m}+\alpha a^{2} \nabla \gamma, \\
\sum_{i} \vec{T}_{i} & =\vec{T}_{\nabla \gamma}+\vec{T}_{f r}=I \vec{\varepsilon}
\end{aligned}
$$


where $m$ and $\vec{v}_{c m}$ are the mass and velocity of the center of mass of the marble, respectively (the change in the mass of marble due to evaporation is neglected) ${ }^{43}$ The second term on the right-hand side in Eq. 1a (namely $\alpha a^{2} \nabla \gamma$ ) is the Marangoni force $^{43}$ The characteristic length $a$ is of the order of magnitude the radius of the marble contact area, shown in Figure 5 (see also ref. 17). The friction force $\vec{F}_{f r}$ mainly comes from the viscous drag ${ }^{42,43}$ which is proportional to the dynamic viscosity of the supporting liquid $\eta ; \alpha$ and $\chi$ are dimensionless coefficients; $\chi=3 \pi$ in the case of Stokes drag of a solid sphere in an unbounded liquid; however it is not well established for floating bodies. The complicated geometry of the vapor layer, depicted schematically in Figure 5, was treated in detail in ref. 62. Now consider Eq. 1b, describing the planar rotation motion of a self-propelled marble: $\vec{T}_{\nabla \gamma}$ and $\vec{T}_{f r}$ are the moments of forces (torques) arising from the gradient of the surface tension (to be treated in detail below) and friction forces, respectively, $I$ is the moment of inertia of the marble about its center of mass and $\vec{\varepsilon}$ is the angular acceleration. The marbles kept their shape practically unchanged in a course of self-propulsion; this observation justifies their treatment as rigid bodies in Eq. $1 \mathrm{~b}$.

Consider first the translation (rectilinear) motion of marbles. As demonstrated in refs. 37,39 and 42 the modulus of the velocity of the center of mass $v_{c m}$ scales as:

$$
v_{c m} \cong \frac{|\Delta \gamma|}{\eta_{w}},
$$

where $\left|\Delta \gamma^{\text {camphor }}\right|$ is the change in the surface tension of water due to the adsorption of camphor and $\eta_{w}$ is the water viscosity. The typical velocity of the center mass was 
established experimentally as $v_{c m}^{\text {camphor }} \approx 10^{-2} \frac{\mathrm{m}}{\mathrm{s}}$ (see Figure 6). It is noteworthy that much larger velocities were registered for marbles driven by alcohol solutions, which were as high as $v_{c m}^{\text {alcohol }} \approx 10^{-1} \frac{m}{s} \cdot{ }^{42,43}$. This difference in the velocities of the center mass may be easily explained qualitatively. Indeed, Eq. 2 yields:

$$
\frac{v_{c m}^{\text {alcohol }}}{v_{c m}^{\text {camphor }}}=\frac{\left|\Delta \gamma^{\text {alcohol }}\right|}{\left|\Delta \gamma^{\text {camphor }}\right|} \cong \frac{40 \frac{\mathrm{mJ}}{\mathrm{m}^{2}}}{4 \frac{\mathrm{mJ}}{\mathrm{m}^{2}}}=10
$$

where $\left|\Delta \gamma^{\text {camphor }}\right| \cong 4 \frac{m J}{m^{2}}$ (see Section 2.4) and $\left|\Delta \gamma^{\text {alcohol }}\right| \cong 40 \frac{m J}{m^{2}}$ (see ref. 56) are the maximal changes in the surface tension of water support due to adsorption of camphor and alcohol respectively, as established experimentally. Thus, we recognize from Eq. 3, that the scaling law supplied by Eq. 2 describes adequately the translation motion of self-propelled liquid marbles driven by soluto-capillary Marangoni flows.

\subsection{Rotational motion of marbles}

The analysis of the rotational motion of marbles is more challenging, ${ }^{39}$ although important from the point of view of microfluidic applications. ${ }^{63,} 64$ Experimental data related to the rotational motion (spinning of marbles) are displayed in Figures 6b, 7, 8b and $\mathbf{8 c}$. Very similar results from both a qualitative and a quantitative point of view were registered for liquid marbles coated by lycopodium or fumed fluorosilica powder. Oscillations of the modulus of the velocity of the center mass $v_{c m}$ and angular velocity $\omega$ of spinning were registered for both types of marble (see Figures 6, 8). Oscillation of the speed of the self-propelled BelousovZhabotinsky droplets was reported recently in ref. 65. However in the experimental situation reported in ref. 65 , the oscillations of velocity reflected an oscillating nature 
of the Belousov-Zhabotinsky chemical reaction occurring within the droplet. In our case, it is reasonable to relate the oscillations of the linear and angular velocities to the oscillations of the surface tension of a liquid support similar to those registered and reported in ref. 66, in which self-propelled motion of an alcohol droplet placed on the water surface and driven by Marangoni soluto-capillary flows was investigated. Moreover, the jump in the surface tension driving the droplets was established in ref. 66 as $c a .|\Delta \gamma| \cong 1 \frac{m J}{m^{2}}$, which is close to that reported in Section 2.4 .

First of all, notice that the modulus of the velocity of the center mass of marbles $v_{c m}$ changes in antiphase with the angular velocity $\omega$ (i.e. one increases whilst the other decreases over time), as it is follows from experimental data presented in Figure 6. This is clear from the "momentary" energy conversation (i.e. within the "small" time span when the friction forces have no chance to change the total energy of a marble; the characteristic time of viscous friction will be discussed below). Indeed, the total energy $E$ of a translationally displaced spinning rigid body is given by: $E=\frac{m v_{c m}^{2}}{2}+\frac{I_{c m} \omega^{2}}{2}$, where $m$ and $I_{c m}$ are the mass and moment inertia of a body about the axis running along the center of mass. Negative values of $\omega$ seen in Figure 8c evidence the change of the rotation direction, observed experimentally.

We first address qualitatively the mechanism of rotation (spinning), illustrated with Figure 9. When evaporation of camphor is spontaneously increased in the direction $-x$, coinciding with the horizontal axis of symmetry of the floating marble passing over its center of mass, the field of Marangoni soluto-capillary stresses (which is symmetrical with respect to the $x$ axis) is created (as shown with black arrows in Figure 9). In this case, the pure translational motion of a marble is expected. In the situation when the direction of dominating evaporation of camphor from a marble 
(shown by the red arrow in Figure 9) does not coincide with the horizontal axis symmetry of a marble, the asymmetric field of Marangoni stresses is expected (as depicted in Figure 9B), giving rise to the torque rotating a marble. The accurate solution of the equations describing the rotation of marbles presents severe mathematical difficulties; thus, we consider simplified scaling laws describing the rotational motion. If the friction is neglected, the maximal angular acceleration of marbles $\varepsilon_{\max }$ may be roughly estimated from the Newtonian law related to the rotation of marbles (see Eq. 1b):

$$
\vec{T}_{\nabla \gamma} \cong\left|\Delta \gamma_{y}\right| a^{2} \cong I_{c m} \varepsilon_{\max }=\frac{2}{5} m R^{2} \varepsilon_{\max }
$$

where $\left|\Delta \gamma_{y}\right|$ is the change of the modulus of the surface tension across the contact area of a marble created in the $y$-direction due to the asymmetric evaporation of camphor, shown in Figure 9B; $m$ and $R$ are the mass and radius of a marble respectively. Considering $m \cong \rho \frac{4}{3} \pi R^{3}$ (with $\rho$ as the density of the aqueous camphor solution, which is close to that of water) yields:

$$
\left|\Delta \gamma_{y}\right| a^{2} \cong \rho R^{5} \varepsilon_{\max }
$$

The numerical factors in Eq. 5 are omitted for the sake of simplicity. For establishing the scaling law governing the rotation of marbles we also need the equation governing the inter-relation between the geometrical parameters of a marble, namely its radius $R$ and the radius of the contact area $a$ (see Figure 5). For floating marbles this inter-relation turns out to be complicated and non-trivial as demonstrated in ref. 40. For the purpose of a very rough estimation we adopt scaling laws, derived for liquid marbles placed on a solid support in ref. 17, namely:

$$
a \sim R^{2} l_{c a}^{-1} \text { for } R>l_{c a}
$$




$$
a \sim R^{3 / 2} l_{x a}^{-1 / 2} \text { for } R<l_{c a}
$$

where $l_{c a}$ is the so-called capillary length. ${ }^{67,}{ }^{68}$ Substituting Eqs. 6 into Eq. 5 supplies the following scaling laws:

$$
\begin{gathered}
\varepsilon_{\max } \sim \frac{\left|\Delta \gamma_{y}\right|}{\rho R l_{c a}^{2}} \sim V^{-1 / 3} \text { for } R>l_{c a} \\
\varepsilon_{\max } \sim \frac{\left|\Delta \gamma_{y}\right|}{\rho R^{2} l_{c a}} \sim V^{-2 / 3} \text { for } R<l_{c a}
\end{gathered}
$$

Actually, the radii of marbles used in our investigation were close to the capillary length; thus, the choice of a proper scaling law describing the rotation became $\quad$ problematic. However, assuming $\left|\Delta \gamma_{y}\right| \cong 1.0 \frac{\mathrm{mJ}}{\mathrm{m}^{2}} ; \rho \cong 1.0 \times 10^{3} \frac{\mathrm{kg}}{\mathrm{m}^{3}} ; R \cong 0.001 \mathrm{~m} ; \mathrm{l}_{c a} \cong 0.003 \mathrm{~m}$ yields for $\quad$ a crude estimation of the maximal angular acceleration of a rotating marble $\varepsilon_{\max } \sim$ $3.0 \times 10^{2} \frac{\mathrm{rad}}{\mathrm{s}^{2}}$ (as calculated with Eq. 7b), which coincides within an order of magnitude with the experimental findings established for the rotational motion of marbles (the experimental data extracted from the analysis of the rotational motion of the marbles supplied for $\varepsilon_{\max }$ the value of $c a .1 .0 \times 10^{2} \frac{\mathrm{rad}}{\mathrm{s}^{2}}$; consider that the numerical factors are omitted in Eq. 7b). The presented estimation completely neglects friction. Thus, it supplies the maximal value of the angular acceleration.

Eqs. 7 predict that the maximal angular acceleration of marbles is expected to decrease weakly with the radius (volume) of marbles. Indeed, the values of the angular acceleration of marbles averaged over their trajectory decreased with their volume, as shown in Figure 10. It is as also noteworthy that the superposition of the rectilinear and rotational motion (i.e. spinning) was also observed in specific 
experiments performed with liquid marbles filled with aqueous solutions of alcohols $\mathrm{s}^{42}$, and it is reasonable to relate the origin of spinning to the asymmetry of the field of Marangoni stresses, depicted schematically in Figure 9B, and described qualitatively by Eqs. 4-7. Recall that the breaking of symmetry of liquid marbles due to evaporation of volatile compounds is a spontaneous process and both pure translational (as shown in Figure 9a) and superposition of the translational and rotational motions (as depicted in Figure 9b) are possible.

Now we address the characteristic period of oscillations of the linear and angular velocities, which is seen from Figures $\mathbf{6}$ and $\mathbf{8}$ to be of the order of magnitude around $10 s$ for both kinds of marbles (it is noteworthy this time scale is close to that of the period of oscillations of the surface tension reported in ref. 66). The characteristic period of oscillations of the linear and angular velocities was performed with the Fourier analysis of the data represented in Figures 6 and 8. The Fourier analysis indicated the pronounced dominating frequencies appearing in the spectra (see Supplementary Material), which allowed the calculation of the period of oscillation of the linear and angular velocities. The characteristic period of oscillations was established as: $t \cong 10-20 \mathrm{~s}$. What is the physical reason responsible for this value?

Until now, we completely neglected the friction forces; however, this time scale is easily identified with the characteristic time of friction $\tau_{f r}$, arising from Eq. 1 and discussed in detail in ref. 43. Considering the results reported in ref. 43 and Eq. $7 \mathrm{a}$ yields:

$$
\tau_{f r} \cong \frac{4 \pi}{3} \frac{R^{3}}{\chi a \eta_{w}} \cong \frac{4 \pi}{3} \frac{\rho R l_{c a}}{\eta_{w}}
$$


Assuming: $\chi \cong 1 ; \eta_{w} \cong 10^{-3} \mathrm{~Pa} \times s ; \rho \cong 10^{3} \frac{\mathrm{kg}}{\mathrm{m}^{3}} R \cong 0.001 \mathrm{~m} ; l_{c a} \cong 0.003 \mathrm{~m}$, supplies for the characteristic friction time the estimation $\tau_{f r} \cong 10 \mathrm{~s}$, which coincides qualitatively with the period of oscillation of the linear and angular velocities as established by Fourier analysis of the experimental data, represented in Figures $\mathbf{6}$ and $\mathbf{8}$ (see also the Supplementary Material).

Collisions of rotating marbles with the skirting of the Petri dish also deserve consideration. It turned out that these collisions take place in a random way: sometimes liquid marbles changed the direction of their rotation after the collision and sometimes continued to rotate in the same direction. We plan to study these fascinating collisions in our future research.

\subsection{Role of thermal Marangoni flows in the self-propulsion of marbles}

Co-occurrence of soluto- and thermo-capillary Marangoni flows is often observed under self-propulsion. ${ }^{14,69}$ Thermal imaging of the self-propulsion of liquid marbles containing saturated aqueous solutions of camphor (see Section 2.5) showed that the temperature field formed at the liquid support surface under self-propulsion was not uniform, and the maximal change in temperature across the liquid/vapor interface was established as $\Delta T_{\max } \cong 0.25 \mathrm{~K}$.

The corresponding change in the surface tension is estimated as: $\left|\Delta \gamma_{T}\right| \cong\left|\frac{\partial \gamma(T)}{\partial T}\right| \Delta T \cong 0.17 \frac{m J}{m^{2} K} \times 0.25 K \cong 4 \times 10^{-2} \frac{m J}{m^{2}}$, which is two orders of magnitude smaller than that due to the adsorption of camphor (see Section 2.4). Hence it is reasonable to suggest that the soluto-capillary Marangoni flows in the selfpropulsion were decisive. It should be emphasized that the registered thermal Marangoni flows supported self-propulsion. ${ }^{14,69}$ 


\section{CONCLUSIONS}

Marangoni soluto-capillary flows give rise to the self-propulsion of a variety of physical objects ${ }^{2-7}, 58-60$, thus demonstrating a potential for microfluidic applications. ${ }^{51}$ The effect of self-propulsion may be strengthened by the Leidenfrost effect, suppressing the friction forces. ${ }^{35-40,49} \mathrm{We}$ report the self-propulsion of liquid marbles ${ }^{17-24}$ filled by saturated aqueous solutions of camphor and coated by hydrophobic lycopodium or fumed fluorosilica particles. The self-propulsion is due to the Marangoni soluto-capillary flow inspired by the spontaneous evaporation of camphor from marbles (breaking the symmetry of the experimental situation) followed by its adsorption by the water support. The maximal change of the surface tension of water owing to the adsorption of camphor was established experimentally as approximately $4.0 \frac{\mathrm{mJ}}{\mathrm{m}^{2}}$. The observed self-propulsion exhibited the superposition of translational and rotational motions. The velocities of the center of mass and the angular velocity of marbles' spinning demonstrated oscillations occurring in antiphase. It is plausible to relate these oscillations to the oscillations of the surface tension of the liquid support. The characteristic period of these oscillations coincides with the time scale of viscous friction, damping the self-propulsion.

A qualitative model and the scaling laws describing the self-propulsion are proposed. The model delineated adequately the translation (rectilinear) and rotational motions observed under the self-locomotion. The model predicted a weak decrease in the angular acceleration of marbles with their volume. This prediction was validated experimentally, i.e. the values of the angular acceleration of marbles averaged over their trajectory decreased with their volume. Thermal Marangoni flows ${ }^{14,69}$ supported the self-propulsion; however, the role of soluto-capillary flows was decisive. The 
reported self-propulsion motion of liquid marbles demonstrates a potential for microfluidic applications ${ }^{51}$ and targeted drug delivery. ${ }^{70}$

\section{Notes}

The authors declare no competing financial interest.

\section{ACKNOWLEDGEMENTS}

The authors are indebted to Mr. Victor Multanen for marbles' imaging. Acknowledgement is made to the donors of the Israel Ministry of Absorption for the partial support of the scientific activity of Dr. Mark Frenkel. The study was financially supported by the Ministry of Education and Science of the Russian Federation, Agreement No. 14.607.21.0160 (RFMEFI60716X0160).

\section{SUPPORTING INFORMATION AVAILABLE}

Three movies showing self-propulsion of $10 \mu \mathrm{L}$ and $50 \mu \mathrm{L}$ liquid marbles containing saturated aqueous camphor solutions and coated with lycopodium and the movie showing self-propulsion of $10 \mu \mathrm{L}$ liquid marble coated with fumed fluorosilica particles are supplied. The marbles are marked with carbon black particles.

One thermographic movie showing self-propulsion of $20 \mu \mathrm{L}$ liquid marble containing saturated aqueous camphor solutions and coated with lycopodium.

\section{AUTHOR INFORMATION}

*Corresponding Author: E-mail: edward@,ariel.ac.il 


\section{REFERENCES}

(1) Ryazantsev, Y,S.; Velarde, M. G.; Rubio, R. G.; Guzmán, E.; Ortega, F.; López, P. Thermo- and soluto-capillarity: Passive and active drops. Adv. Colloid Interface Sci. 2017, 247, 52-80.

(2) Sano, O.; Kutsumi, K.; Watanabe, N. Surface tension driven oscillatory motion of a mercury drop in the presence of concentration gradient of $\mathrm{K}_{2} \mathrm{Cr}_{2} \mathrm{O}_{7}$ in $\mathrm{HNO}_{3}$ solution. J. Phys. Soc. Jpn. 1995, 64, 1993-1999.

(3) Snezhko, A.; Belkin, M.; Aranson, I. S.; Kwok, W.-K. Self-assembled magnetic surface swimmers. Phys. Rev. Lett. 2009, 102, 118103.

(4) Paxton, W. F.; Sen, A.; Mallouk, Th. E. Motility of catalytic nanoparticles through self-generated forces. Chemistry - A European J. 2005, 11, 6462-6470.

(5) Mitsumata, T.; Gong, J. P.; Osada, Y. Shape memory functions and motility of amphiphilic polymer gels. Polym. Adv. Technol. 2001, 12, 136-150.

(6) Osada, Y.; Gong, J. P.; Uchida, M.; Isogai, N. Spontaneous motion of amphoteric polymer gels on water. Jpn. J. Appl. Phys. 1995, 34, L511.

(7) Singh, V.; Wu, C.-J.; Sheng, Y-J.; Tsao, H-K. Self-propulsion and shape restoration of aqueous drops on sulfobetaine silane surfaces. Langmuir 2017, 33, 6182-6191.

(8) Daniel, S.; Chaudhury, M. K.; Chen, J. C. Fast drop movements resulting from the phase change on a gradient surface. Science 2001, 291, 633-636.

(9) Daniel, S.; Sircar, S.; Gliem, J.; Chaudhury, M. K. Ratcheting motion of liquid drops on gradient surfaces. Langmuir 2004, 20, 4085-4092. 

Li, J.; Hou, Y.; Liu, Y.; Hao, Ch.; Li, M.; Chaudhury, M. K.; Yao, Sh.; Wang, Z. Directional transport of high-temperature Janus droplets mediated by structural topography. Nature Physics 2016, 12, 606-612.

(11) Zheng, X.-P.; Zhao, H.-P.; Gao, L.-T.; Liu, J.-L.; Yu, Sh-W.; Feng, X.-Q. Elasticity-driven droplet movement on a microbeam with gradient stiffness: A biomimetic self-propelling mechanism. J. Colloid Interface Sci. 2008, 323, 133-140.

(12) Semenov, S.; Starov, V. M.; Velarde, M. G.; Rubio, R.G. Droplets evaporation: Problems and solutions. Eur. Phys. J. Special Topics 2011, 197, 265 278.

(13) Nepomnyashchy, A. A.; Velarde, M. G.; Colinet, P. Interfacial Phenomena and Convection; Boca Raton \& London. Chapman \& Hall/CRC Press, 2002.

(14) Krechetnikov, R. Thermodynamics of chemical Marangoni-driven engines, Soft Matter 2017, 13, 4931-4950

(15) Colinet, P.; Legros, J. C.; Velarde, M. G. Nonlinear Dynamics of SurfaceTension-Driven Instabilities; Wiley: Berlin, 2001.

(16) Starov, V.; Sefiane, Kh. On evaporation rate and interfacial temperature of volatile sessile drops. Colloids Surf. A 2009, 333, 170-174.

(17) Aussillous, P.; Quéré, D. Liquid marbles. Nature 2001, 411, 924-927.

(18) Bormashenko, E. Liquid marbles, elastic nonstick droplets: from mini-reactors to self-propulsion. Langmuir 2017, 33, 663-669.

(19) Binks, B. P.; Murakami, R. Phase inversion of particle-stabilized materials from foams to dry water. Nature Mater. 2006, 5, 865-869.

(20) Zang, D.; Chen, Zh.; Zhang, Y.; Lin, K.; Geng, X.; Binks, B. P. Effect of particle hydrophobicity on the properties of liquid water marbles. Soft Matter 2013, 9, 5067-5073. 
(21) Janardan, M.; Panchagnula, M.; Bormashenko, E. Liquid marbles: Physics and applications. Sadhana, 2015, 40, 653-671.

(22) Tian, J.; Arbatan, T.; Li, X.; Shen, W. Liquid marble for gas sensing. Chem. Commun. 2010, 46, 4734-4736.

(23) Dupin, D.; Armes, St. P.; Fujii, S. Stimulus-responsive liquid marbles. J. Am. Chem. Soc. 2009, 131, 5386-5387.

(24) Liu, Zh.; Fu, X.; Binks, B. P.; Shum, H. Ch. Coalescence of electrically charged liquid marbles. Soft Matter 2017, 13, 119-124

(25) Bhosale. P. S.; Panchagnula, M; Stretz, H. A. Mechanically robust nanoparticle stabilized transparent liquid marbles. Appl. Phys. Lett. 2008, 93, 034109.

(26) McHale, G. Newton, M. I. Liquid marbles: principles and applications. Soft Matter 2011, 7, 5473-5481.

(27) Tosun, A.; Erbil, H. Y. Evaporation rate of PTFE liquid marbles. Appl. Surf. Sci. 2009, 256,1278-1283.

(28) Dandan, M.; Erbil, H. Y. Evaporation rate of graphite liquid marbles: comparison with water droplets. Langmuir 2009, 25, 8362-8367.

(29) Paven, M; Mayama, K.; Sekido, Y.; Butt, H-J.; Nakamura, Y.; Fujii, S. Lightdriven delivery and release of materials using liquid marbles. Adv. Funct. Mater. 2016, 26, 3199-3206.

(30) Bormashenko, Y.; Pogreb, R.; Gendelman, O. Janus droplets: liquid marbles coated with dielectric/semiconductor particles. Langmuir 2011, 27, 7-10.

(31) Ch.-H. Ooi, N.-Tr. Nguyen, Manipulation of liquid marbles. Microfluidics \& Nanofluidics, 2015, 19, 483-495.

(32) Khaw, M. K.; Ooi, Ch. H.; Mohd-Yasin, F.; Nguyen, A. V.; Evans, G. M; Nguyen, N-Tr. Dynamic behaviour of a magnetically actuated floating liquid marble. 
Microfluidics \& Nanofluidics, 2017, 21, 110.

(33) Bormashenko, E.; Pogreb, R.; Bormashenko, Y.; Musin, A.; Stein, T. New investigations on ferrofluidics: Ferrofluidic marbles and magnetic-field-driven drops on superhydrophobic surfaces. Langmuir 2008, 24, 12119-12122.

(34) Ooi, Ch. H.; Bormashenko, Ed.; Nguyen, A. V.; Evans, G. M.; Dao, D. V.; Nguyen, N.-Tr. Evaporation of ethanol-water binary mixture sessile liquid marbles. Langmuir 2016, 32, 6097-610.

(35) Bormashenko, E.; Bormashenko, Y.; Musin, A.; Barkay, Z. On the mechanism of floating and sliding of liquid marbles. ChemPhysChem. 2009, 10, 654656.

(36) Biance, A-L.; Clanet, C.; Quéré, D. Leidenfrost drops. Phys. Fluids 2003, 15, 1632.

(37) Quéré, D. Leidenfrost dynamics. Ann. Rev. Fluid Mech. 2013, 45, 197-215.

(38) Burton, J. C.; Sharpe, A. L.; van der Veen, R. C.; Franco, A. Nagel, S. R. The Geometry of the vapor layer under a Leidenfrost drop. Phys. Rev. Lett. 2012, 109, 074301.

(39) Mrinal, M.; Wang. X.; Luo, C. Self-rotation-induced propulsion of a Leidenfrost drop on a ratchet. Langmuir 2017, 33, 6307-6313.

(40) Wong, Cl. Y. H.; Adda-Bedia, M.; Vella, D. Non-wetting drops at liquid interfaces: from liquid marbles to Leidenfrost drops. Soft Matter 2017, DOI: 10.1039/C7SM00990A

(41) Janssens, S. D.; Koizumi, S.; Fried, E. Behavior of self-propelled acetone droplets in a Leidenfrost state on liquid substrates. Phys. Fluids 2017, 29, 032103. 
(42) Bormashenko, Ed.; Bormashenko, Ye.; Grynyov, R.; Aharoni, H.; Whyman, G; Binks, B, P. Self-Propulsion of liquid marbles: Leidenfrost-like levitation driven by Marangoni flow. J. Phys. Chem. C 2015, 119, 9910-9915.

(43) Ooi, Ch. H.; Nguyen, A.; Evans, G. M.; Gendelman, O.; Bormashenko, Ed.; Nguyen N.-Tr. A floating self-propelling liquid marble containing aqueous ethanol solutions. RSC Adv. 2015, 5, 101006-101012.

(44) Kavokine, N.; Anyfantakis, M.; Morel, M.; Rudiuk, S.; Bickel, Th.; Baigl, D. Light-driven transport of a liquid marble with and against surface flows. Angew. Chem. 2016, 128, 11349-11353.

(45) Nakata, S.; Matsuo K. Characteristic self-motion of a camphor boat sensitive to ester vapor. Langmuir 2005, 21, 982-984.

(46) Nakata, S.; Yoshii, M.; Matsuda, Y.; Suematsu, N. J. Characteristic oscillatory motion of a camphor boat sensitive to physicochemical environment, Chaos 2015, 25, 064610.

(47) Kohira, M. I.; Hayashima, Y.; Nagayama, M.; Nakata, S. Synchronized selfmotion of two camphor boats. Langmuir 2001, 17, 7124-7129.

(48) Nakata, S.; Yamamoto, H. Yuki Koyano, Y.; Yamanaka, O.; Sumino, Y.; Suematsu, N. J.; Kitahata, H.; Skrobanska, P.; Gorecki, J. Selection of the rotation direction for a camphor disk resulting from chiral asymmetry of a water chamber. $J$. Phys. Chem. B 2016, 120, 9166-9172.

(49) Hayashima, Y.; Nagayama, M.; Nakata, S. A Camphor grain oscillates while breaking symmetry. J. Phys. Chem. B 2001, 105, 5353-5357.

(50) Wong C. Y. H.; Adda-Bedia, M.; Vella, D. Non-wetting drops at liquid interfaces: from liquid marbles to Leidenfrost drops. Soft Matter 2017, 13, 5250-5260. 
(51) Nguyen, N-Tr.; Hejazian, M.; Ooi, Vh. H.; Kashaninejad, N. Recent advances and future perspectives on microfluidic liquid handling. Micromachines 2017, 8, 186. (52) Zhao, Y.; Hu, Z.; Parhizkar, M.; Fang, J.; Wang, X.; Lin, T. Magnetic liquid marbles, their manipulation and application in optical probing. Microfluid. Nanofluid. 2012, 13, 555-564.

(53) Li, H.; Liu, P.; Kaur, G.; Yao, X.; Yang, M. Transparent and gas-permeable liquid marbles for culturing and drug sensitivity test of tumor spheroids. $A d v$. Healthcare Mater. 2017, 3, DOI: 10.1002/adhm.201700185

(54) Binks, B. P.; Tyowua, A. T. Influence of the degree of fluorination on the behavior of silica particles at air-oil surfaces. Soft Matter 2013, 9, 834-845.

(55) Bormashenko, Ed.; Stein, T.; Pogreb, R.; Aurbach, D. Petal effect on surfaces based on lycopodium: high-stick surfaces demonstrating high apparent contact angles. J. Phys. Chem. C 2009, 113, 5568-5572.

(56) Musin, A.; Grynyov, R.; Frenkel, M.; Bormashenko, Ed. Self-propulsion of a metallic superoleophobic micro-boat. J. Colloid Interface Sci. 2016, 479, 182-188.

(57) Frenkel, M.; Whyman, G.; Shulzinger, T.; Starostin, A.; Bormashenko, Ed. Selfpropelling rotator driven by soluto-capillary Marangoni flows. Appl. Phys. Lett. 2017, $110,131604$.

(58) Moo, J.; Pumera, M. Chemical energy powered nano/micro/macromotors and the environment. Chemistry - A European J. 2015, 21, 58-72.

(59) Ismagilov, R. F.; Schwartz, A.; Bowden, N.; Whitesides G. M. Autonomous movement and self-assembly. Angew. Chem. 2002, 114, 674-676.

(60) Paxton, W. F.; Sundararajan, Sh.; Mallouk, Th. S.; Sen, A. Chemical locomotion. Angew. Chem. 2006, 45, 5420-5429. 
(61) Sharma, R.; Chang, S. T.; Velev, O. D. Gel-based self-propelling particles get programmed to dance. Langmuir 2012, 28, 10128-10135.

(62) Burton, J. C.; Sharpe, A. L.; van der Veen, R. C.; Franco, A.; Nagel, S. R. The geometry of the vapor layer under a Leidenfrost drop. Phys. Rev. Lett. 2012, 109, 074301.

(63) Han, X.; Lee, H. K.; Lee, Y. H.; Ling, X. Y. Dynamic rotating liquid marble for directional and enhanced mass transportation in three-dimensional microliter droplets. J. Phys. Chem. Lett. 2017, 8, 243-249.

(64) Lee, H. K.; Lee, Y. H.; Phan-Quang, G. Ch.; Han, X.; Koh, Ch. Sh. L.; Ling, X. Y. Constructing soft substrate-less platforms using particle-assembled fluid-fluid interfaces and their prospects in multiphasic applications. Chem. Mater. 2017, 29, $6563-6577$.

(65) Suematsu, N. J.; Mori, Y.; Amemiya, T.; Nakata, S. Oscillation of speed of a self-propelled Belousov-Zhabotinsky droplet. J. Phys. Chem. Lett., 2016, 7, 3424 3428.

(66) Oshima, S.; Nomoto, T.; Toyota, T.; Fujinami, M. Surface tension gradient around an alcohol droplet moving spontaneously on a water surface. Anal. Sci. 2014, $30,441-444$.

(67) Bormashenko, Ed. Wetting of Real Surfaces, de Gruyter, Berlin, 2013.

(68) de Gennes, P. G.; Brochard-Wyart, F.; Quéré D. Capillarity and Wetting Phenomena, Springer, Berlin, 2003.

(69) Venerus, D. C.; Simavilla D. N. Tears of wine: new insights on an old phenomenon. Sci. Rep. 2015, 5, 16162.

(70) Patra, D.; Sengupta, S.; Duan, W.; Zhang, H.; Pavlick, R.; Sen, A. Intelligent, self-powered, drug delivery systems. Nanoscale 2013, 5, 1273-1283. 
(a)

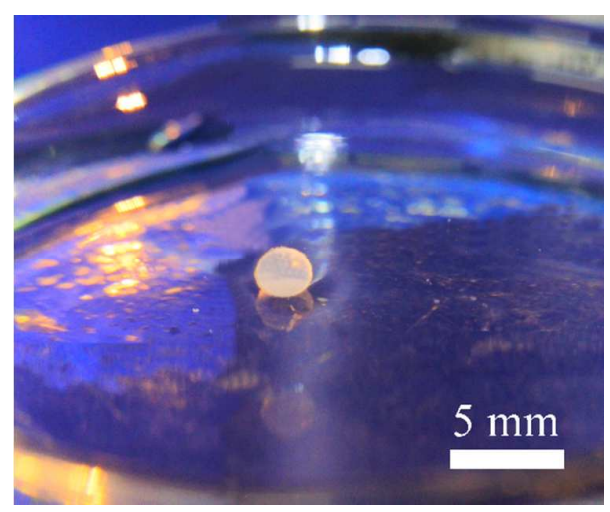

(b)

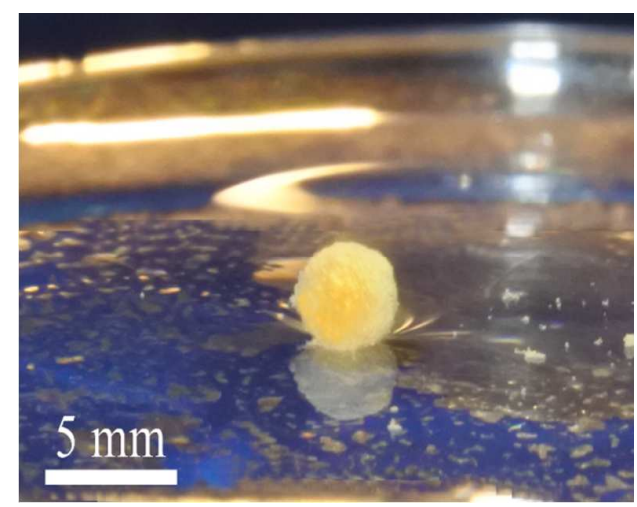

Figure 1. Photos of liquid marbles containing aqueous camphor solutions coated with (a) fumed fluorosilica powder or (b) lycopodium powder floating on a water surface. 


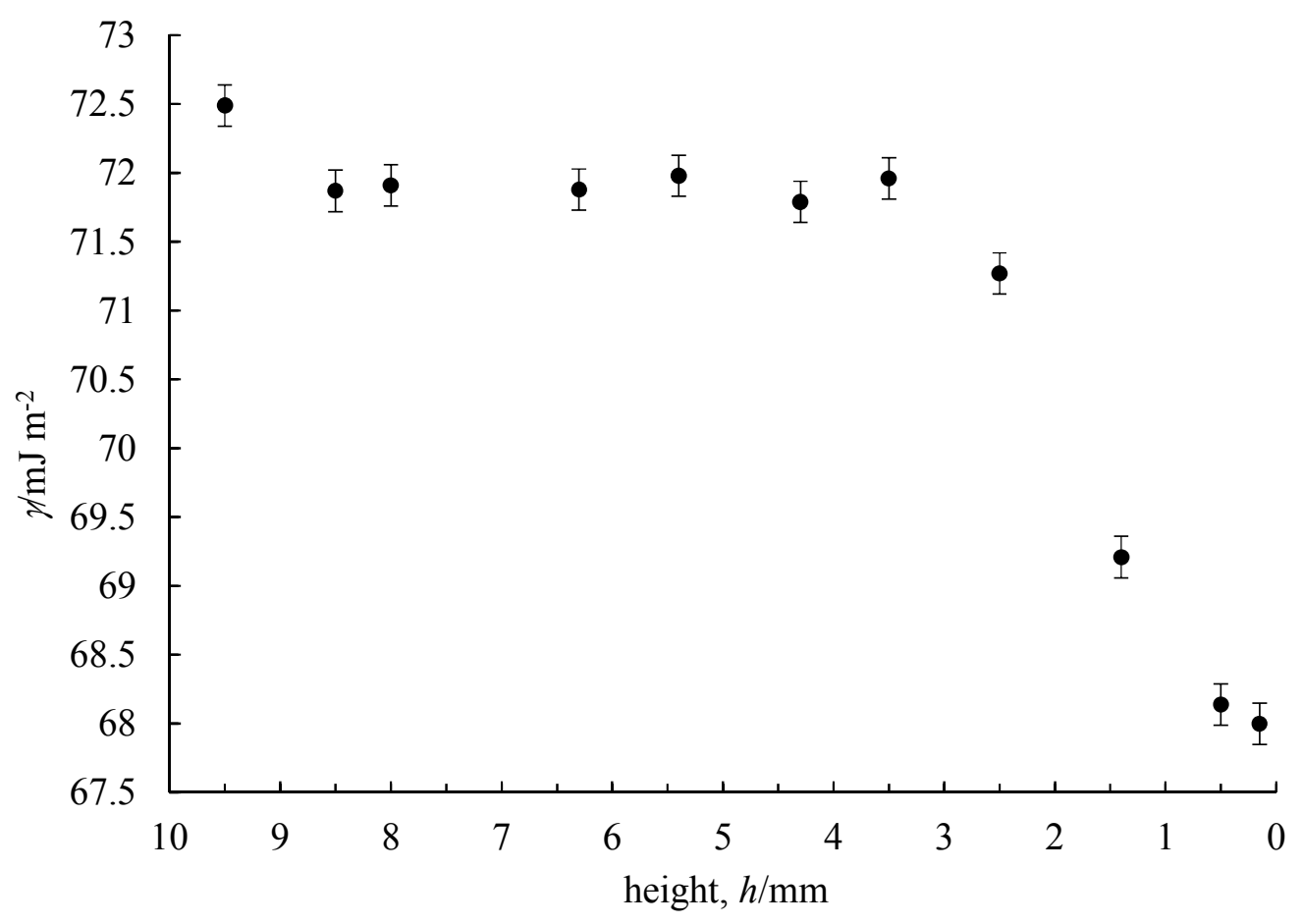

Figure 3. Dependence of the surface tension of a pendant water droplet on the height $h$ (see Figure 2). 


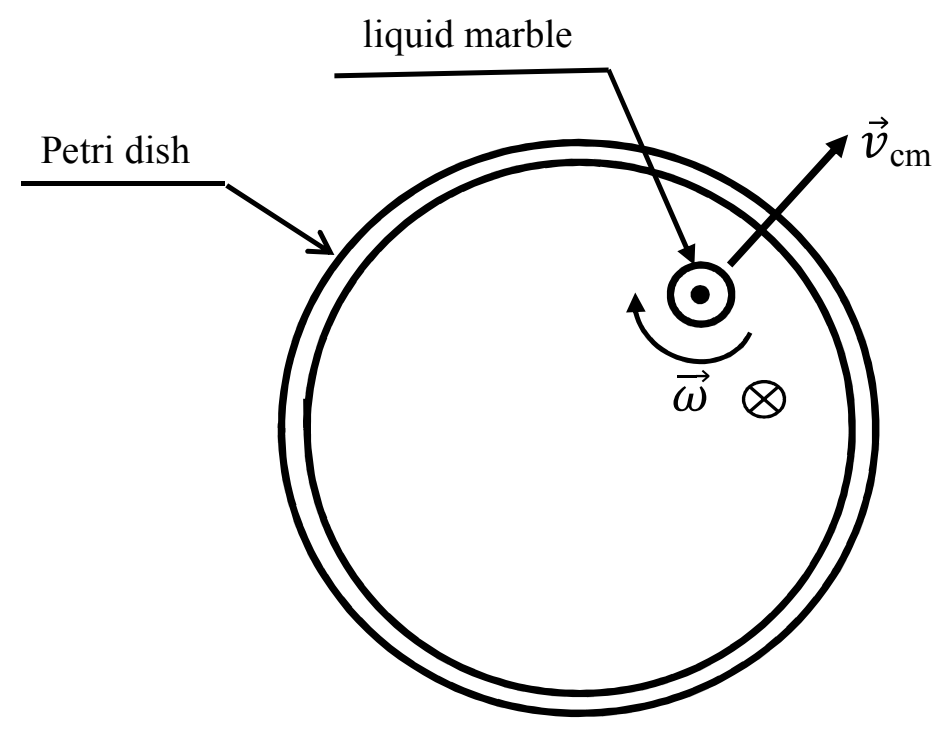

Figure 4. Superposition of the translational and rotational motions observed under self-propulsion of liquid marbles filled with saturated aqueous solutions of camphor. The vectors of the velocity of the center mass $\vec{v}_{c m}$ and angular velocity $\vec{\omega}$ are shown. 


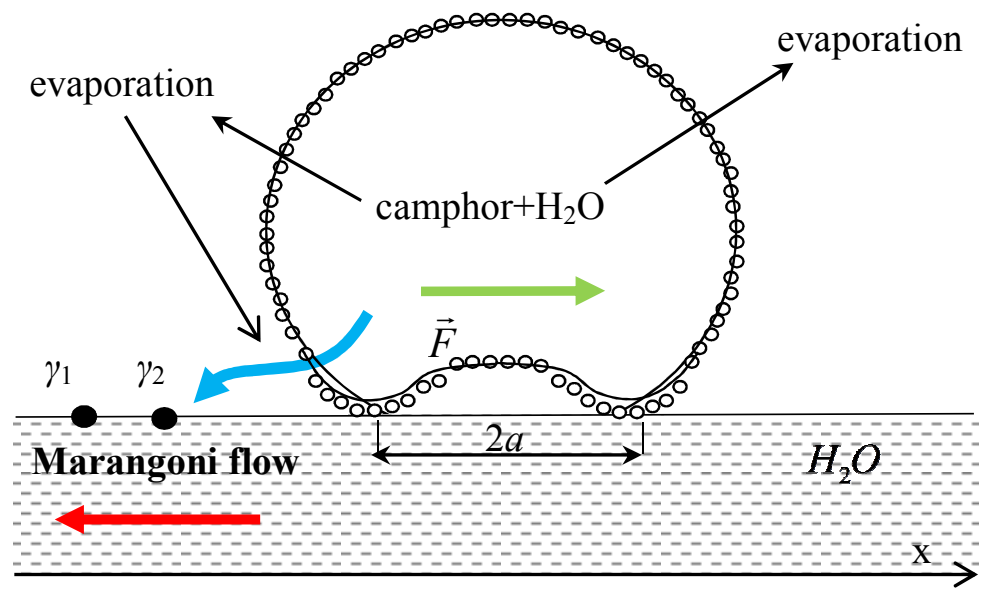

Figure 5. Scheme illustrating the origin of the soluto-capillary Marangoni flow driving liquid marbles containing a saturated aqueous solution of camphor deposited on a water surface. For an explanation of the shape of the vapor layer separating the marble from a liquid support see ref. 62. The blue arrow shows the spontaneous increase of the camphor evaporation from a marble. The red arrow indicates the direction of the Marangoni flow, increasing in turn the evaporation of camphor from the area beneath a marble $\left(\gamma_{1}>\gamma_{2}\right.$ for two points shown on the water surface). 


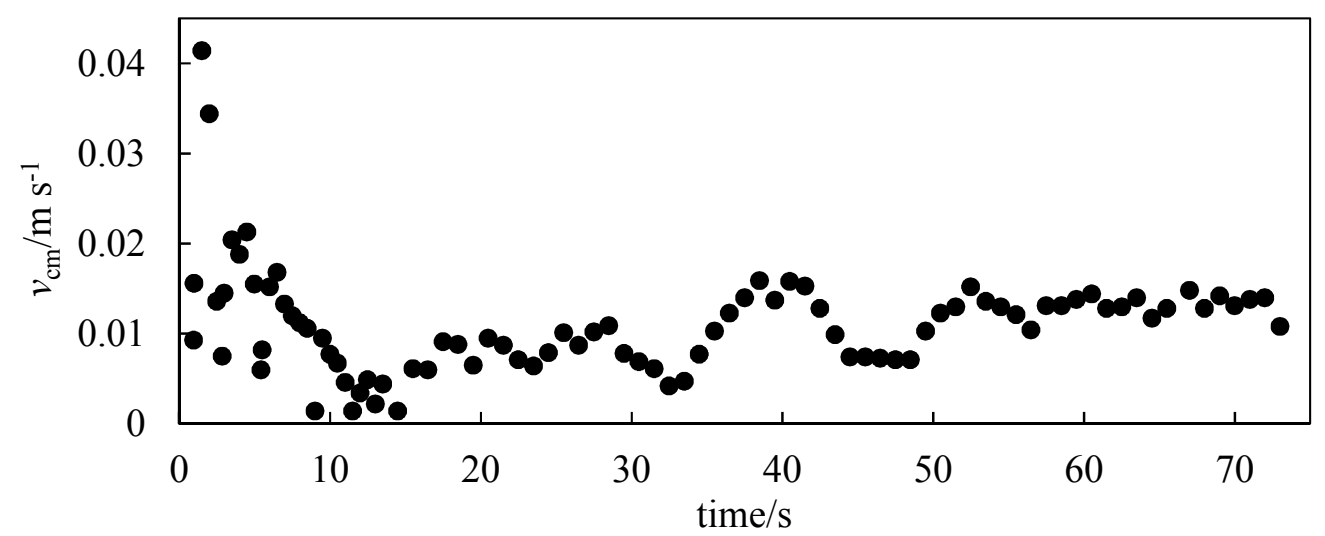

(b)

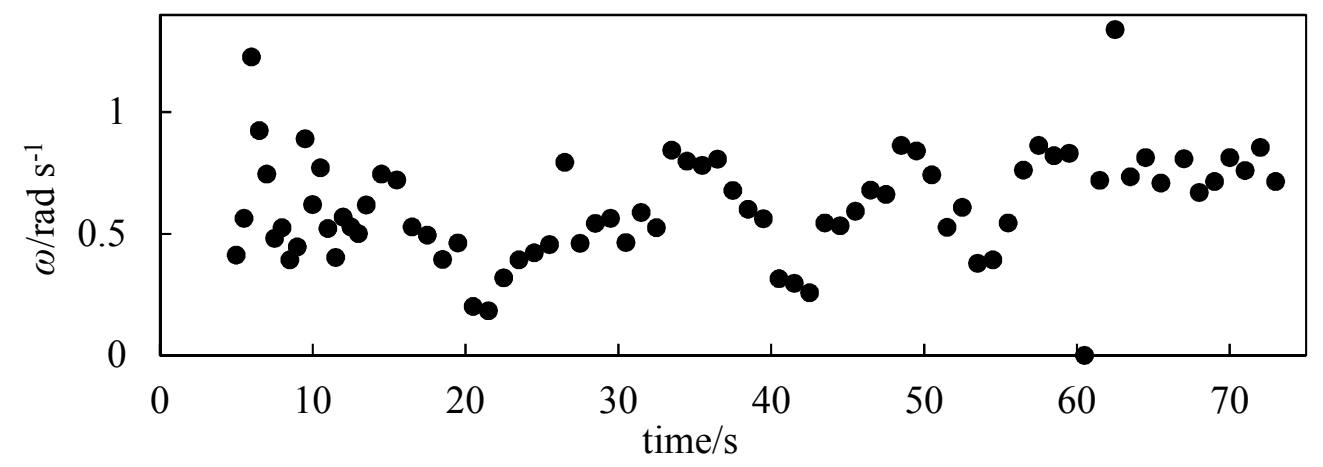

Figure 6. Time dependence of (a) the modulus of the velocity of the center of mass $v_{c m}$ and (b) the angular velocity $\omega$ for $20 \mu \mathrm{L}$ marbles coated with lycopodium powder. 


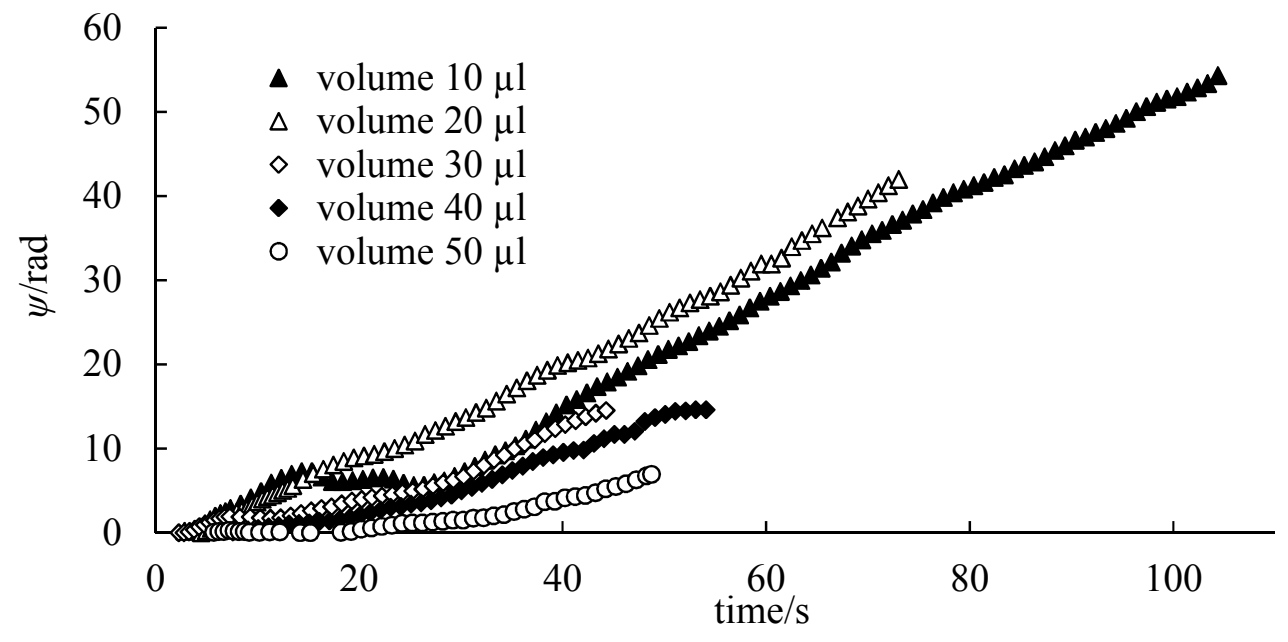

Figure 7. Time dependence of the rotational angle of marbles $\Psi$ (established by observing the rotation of the carbon black marker, see Section 2.3) for marbles of different volume coated with lycopodium $(V=10-50 \mu \mathrm{L})$. 
(a)

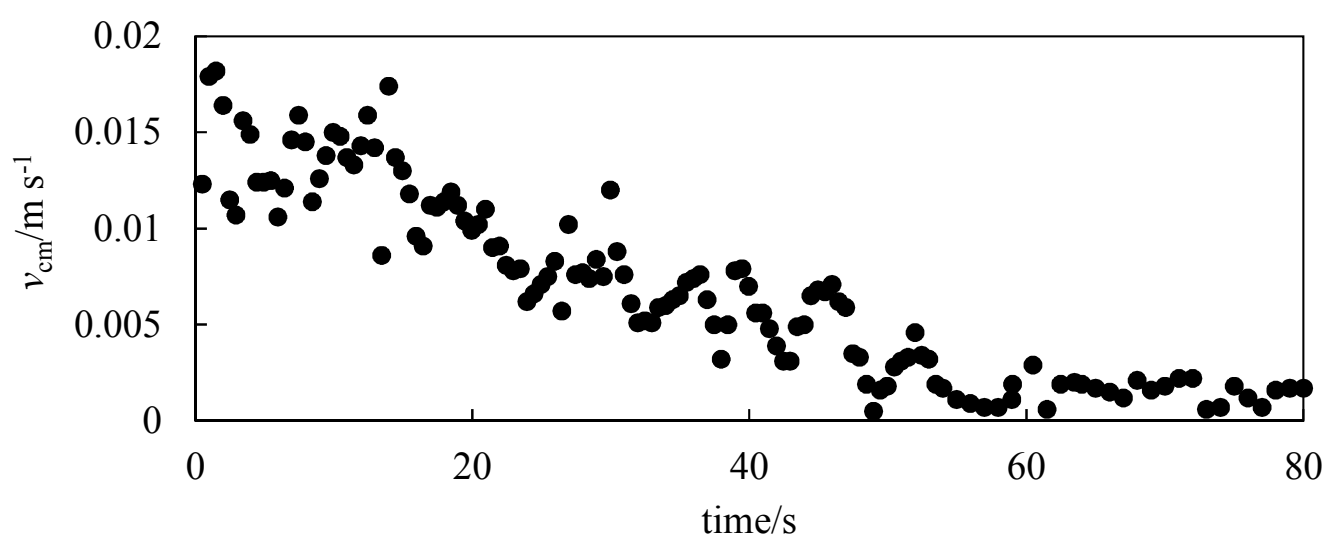

(b)

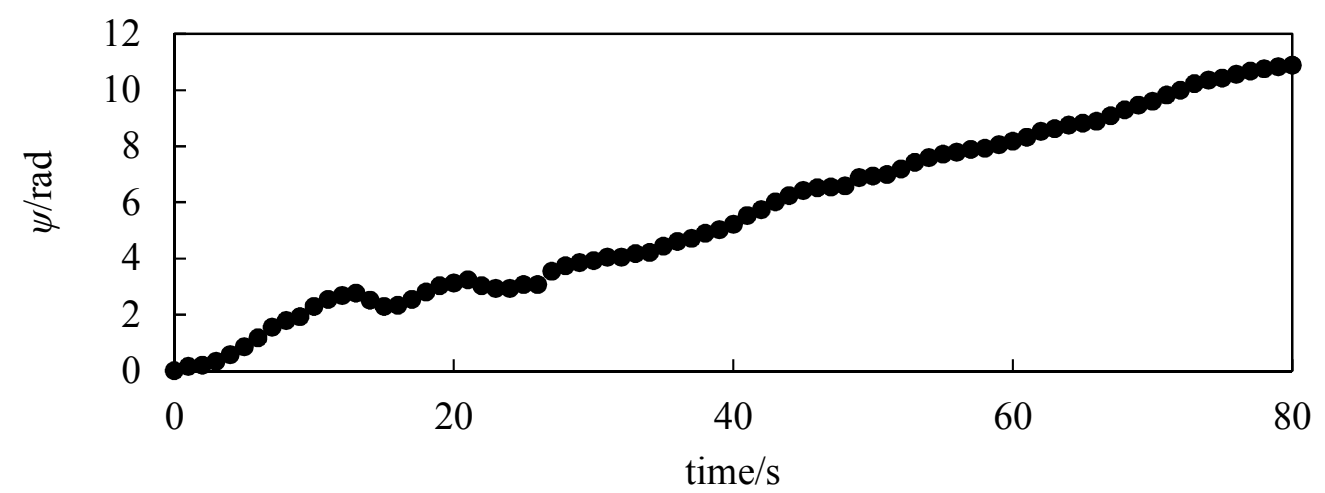

(c)

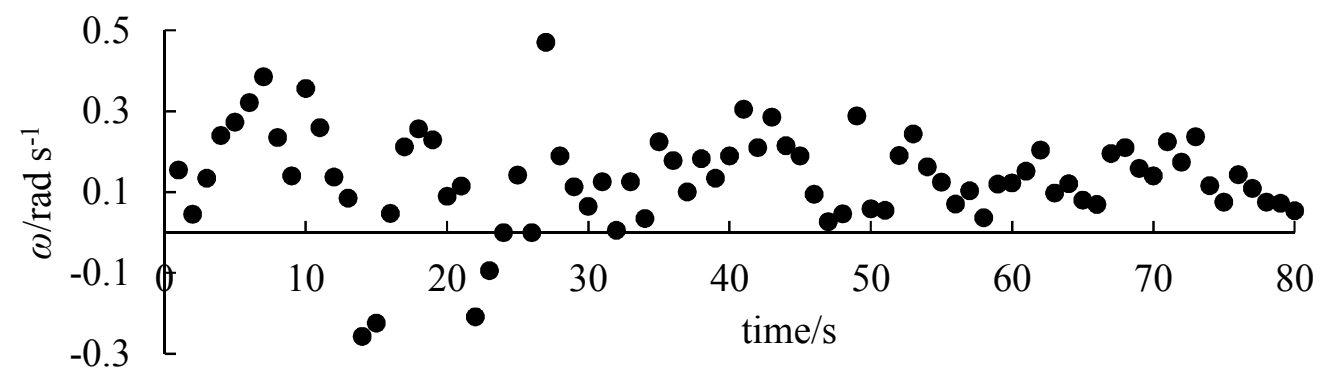

Figure 8. Time dependence of (a) the modulus of the velocity of the center of mass $v_{c m}$, (b) the rotational angle $\Psi$ and (c) the angular velocity $\omega$ of $20 \mu \mathrm{L}$ liquid marbles coated with fumed fluorosilica powder. 
(a)

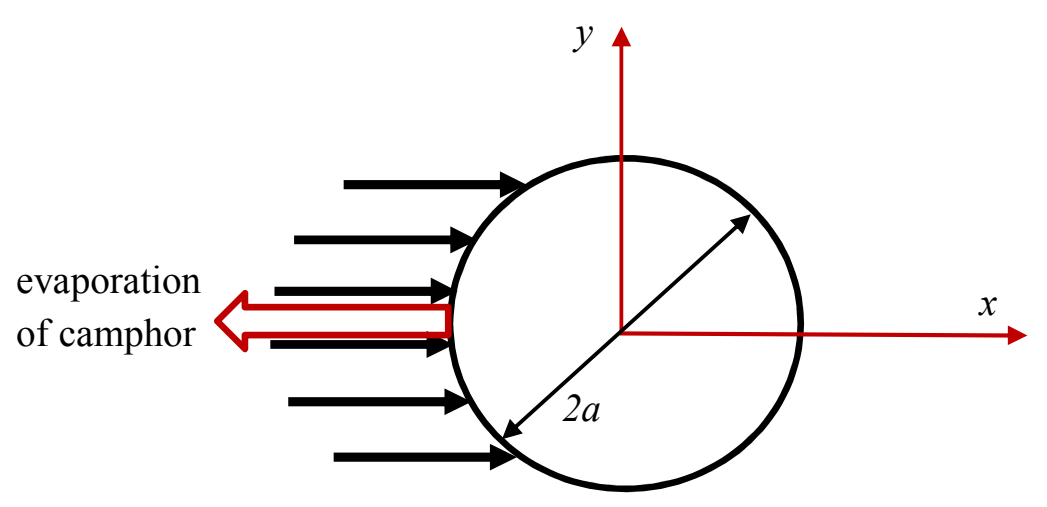

(b)

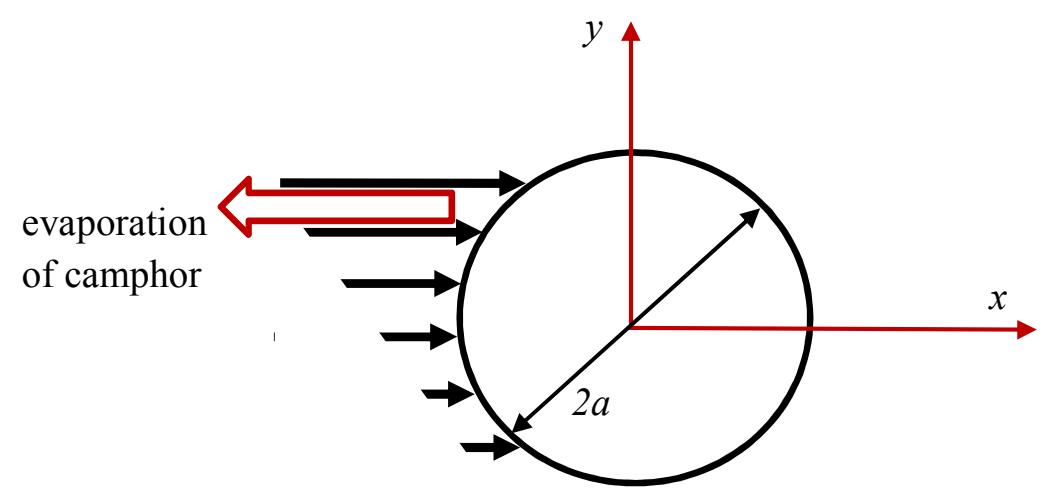

B

Figure 9. The mechanisms giving rise to translational and rotational motions of liquid marbles are displayed. The horizontal cross-section of the area adjacent to the contact area is shown ( $a$ is the radius of the contact area). The red arrow indicates the direction of dominating evaporation of camphor from a marble. Black arrows depict the spatial (plain) distribution of the Marangoni stresses. (a) Distribution of stresses resulting in the pure translational motion of a marble, (b) distribution of stresses giving rise to the superposition of the translational and rotational motions. 


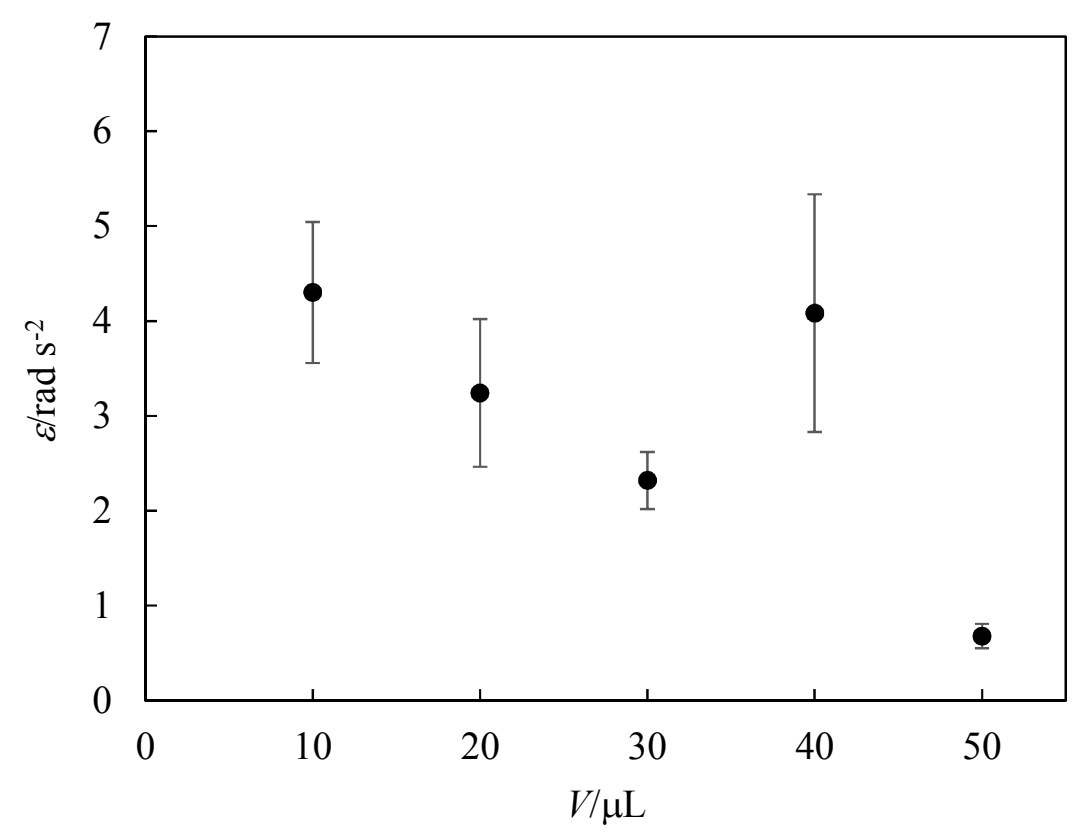

Figure 10. Dependence of the angular acceleration $\varepsilon$ of liquid marbles coated by lycopodium powder (averaged over the piece of trajectory where oscillations of linear and angular velocities were observed) on their volume $V$. 


\section{Page 35 of 35}

Langmuir

1
2
3
4
5
6
7
8
9
10
11
12
13
14
15
16
17
18
19
20
21
22
23
24
25
26
27
28
29
30
31
32
33
34
35
36
37
38
39
40
41
42
43
44
45
56
56
47
48
49
50
51
52

TOC Image

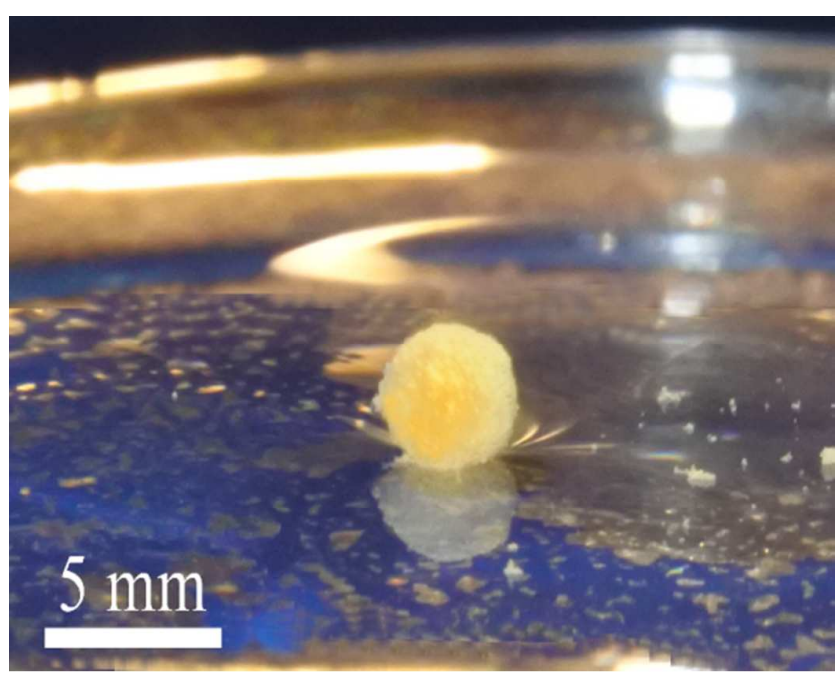

35

ACS Paragon Plus Environment 